\title{
EXPERIMENTAL AND NUMERICAL ANALYSIS OF STICK-SLIP SUPPRESSION WITH THE USE OF LONGITUDINAL TANGENTIAL VIBRATION
}

\author{
Marta Rybiewicz, Pawee Gutowski, Mariusz Leus \\ West Pomeranian University of Technology, Faculty of Mechanical Engineering and Mechatronics, Szczecin, Poland \\ e-mail: marta.rybkiewicz@zut.edu.pl; pawel.gutowski@zut.edu.pl; mariusz.leus@zut.edu.pl
}

\begin{abstract}
The results of experimental tests and numerical simulation analyses of the possibility of partial reduction or entire elimination the stick-slip phenomenon in sliding motion through introduction of longitudinal tangential vibrations to the contact zone of a shifted body and the substrate are shown in the paper. The experimental tests were carried out on a specially designed stand. In the computational model, the dynamic equation of motion of the shifted body was used, and for the friction force description the LuGre model was adopted. A excellent consistency of the experimentally determined results with those calculated with the use of the developed model is obtained.
\end{abstract}

Keywords: stick-slip phenomenon, longitudinal tangential vibrations, LuGre model

\section{Introduction}

The appearance of self-excited relaxation vibrations, called as the stick-slip motion, generated at low slip velocities under the influence of the friction force variation, is a definitely undesirable phenomenon in mechanical devices. The stick-slip phenomenon has an adverse effect on the accuracy of their movement and also causes faster wear of friction pair elements. In particular, the stick-slip phenomenon reduces the accuracy of positioning mobile mechanisms of machine tools and manipulators (Rymuza, 1992; Mfoumou et al., 2019). It occurs when drilling shafts in the mining, gas and oil industry, and significantly reduces the velocity of drilling (Aarsnes et al., 2018; Qiu et al., 2018) and also causes premature wear of drilling tools, considerably lowering the quality of the drilled shaft, which markedly increases costs of drilling (Zhu et al., 2014). The stick-slip motion accelerates wear - abrasion of the movable contact surfaces of friction pairs. For this reason and for the reasons mentioned above, one should strive to limit or completely eliminate this phenomenon.

The theoretical analysis and experimental research of this phenomenon, in terms of possibility of its reduction or entire elimination, have been done by many research centers in the world already in the 1960s. In particular, works of Tolstoi (1967), Tolstoi et al. (1973) and Lenkiewicz (1969) can be pointed out. As a result, various methods have been developed to reduce or completely eliminate this phenomenon, e.g. by selecting relevant materials of friction pairs for the given works conditions and ensuring adequate microgeometry of contacting surfaces in the case of existing materials (Kröger et al., 2008; Kligerman and Varenberg, 2014), or developing new materials for given work conditions reducing or entirely eliminating the possibility of stick-slip motion appearance (Rymuza, 1989, 1992). The possibility of reducing this phenomenon by decreasing normal pressure at the contact or increasing stiffness of the drive system was also analyzed (Rymuza, 1992). A separate issue is the use of appropriate lubricants to eliminate the stick-slip motion (Zuleeg, 2015). 
Experimental tests and theoretical analysis of reduction of this phenomenon by introducing forced vibrations with appropriate parameters into the contact zone were also carried out. The possibility of such a solution was signalled in works of Tolstoi (1967) and Tolstoi et al. (1973) - with regard to normal vibrations, as well as Lenkiewicz (1969) and Broniec and Lenkiewicz (1982) - with regard to tangential to the contact surface vibrations.

An extensive theoretical analysis as well as the experimental tests on the reduction of the stick-slip phenomenon by introducing normal vibrations into the contact zone were carried out recently by Popp and Rudolph (2003, 2004), Kröger et al. (2008), Abdo et al. (2009; 2010) and Abdo and Zaier (2012). The obtained by them experimental tests results indicate that the level of reduction of the stick-slip vibration amplitude by introducing normal vibrations depends on the type of the friction pair material, amplitude and frequency of forced vibration, as well as relative humidity of the environment. Neubauer et al. (2005) and Popp and Rudolph (2003; 2004) described the possibility of stick-slip reduction by active control of normal to the contact surface vibrations using a normal force with a variable amplitude.

Experimental and simulation analysis indicating the possibility of using tangential vibrations to reduce the stick-slip movement were described by Popov et al. (2010) and Teidelt et al. (2012). Similar studies, but in a small range of drive velocity and at low stiffness of the drive system were also described in the work of Jurczyński (2013). There are no works analyzing the impact of changes in drive velocity and drive stiffness on the effectiveness of stick-slip suppression by high-frequency longitudinal tangential vibrations.

Experimental researches of the stick-slip phenomenon require construction of appropriate test stands and they are very time-consuming and labor-intensive. Therefore, they are not very effective, hence it was necessary to develop an appropriate computational model enabling simulation analyzes of this phenomenon, including in particular the influence of various factors on its development or disappearance. Only suitable models of friction can be used in these models.

Comparative analyzes of the possibilities of using different friction models (classic, Karnopp, Dahl) to describe the stick-slip motion and a description of new models developed specifically for modelling this motion (Bristle, Reset Integrator) were presented in the work of Haessig and Friedland (1991), while the works of Canudas de Wit et al. (1995), Olsson (1996) and Åstrom and Canudas de Wit (2008) described the so-called LuGre friction model developed for testing systems in which sliding friction and, in particular, stick-slip vibrations occur.

The purpose of this paper is to present experimental and simulation tests on the impact of the slip velocity and stiffness of the drive on stick-slip vibrations and the possibility of their reduction by introducing longitudinal tangential vibrations of high frequency into the contact zone. Experimental tests were carried out on a specially constructed for this purpose test stand (Leus and Abrahamowicz, 2019), while simulation analyzes were performed using an original computational program created in the Matlab/Simulink environment. In this model, dynamic equations of the shifted body were used to describe motion, and the LuGre dynamic friction model was used to describe the friction force.

\section{Test stand}

A block diagram of the measuring and data handling system is shown in the Fig. 1 while in Fig. 2 a photo of the mechanical part of the test stand is shown. The friction pair consists of shifted upper specimen 1 and vibrating saddle 2. The upper specimen is set to the sliding motion by a drive system with spring 5 of strictly specified stiffness. The lower one can be excited into a high frequency tangential vibration at any time during sliding movement of the upper specimen. These vibrations are generated by piezoelectric actuator 3 . Continuous measurement of the drive force is carried out by ring dynamometer 4 . Acceleration of the lower specimen is measured by 
two accelerometers 6. Displacements of the upper specimen and drive system are measured by two displacement sensors 7.

Both upper 1 and lower specimen 2, which form the tested friction pair, are made of structural steel 45 . The surface roughness of both samples are determined by the parameter $R_{a}$ which ranges from $0.80 \mu \mathrm{m}$ to $0.95 \mu \mathrm{m}$.
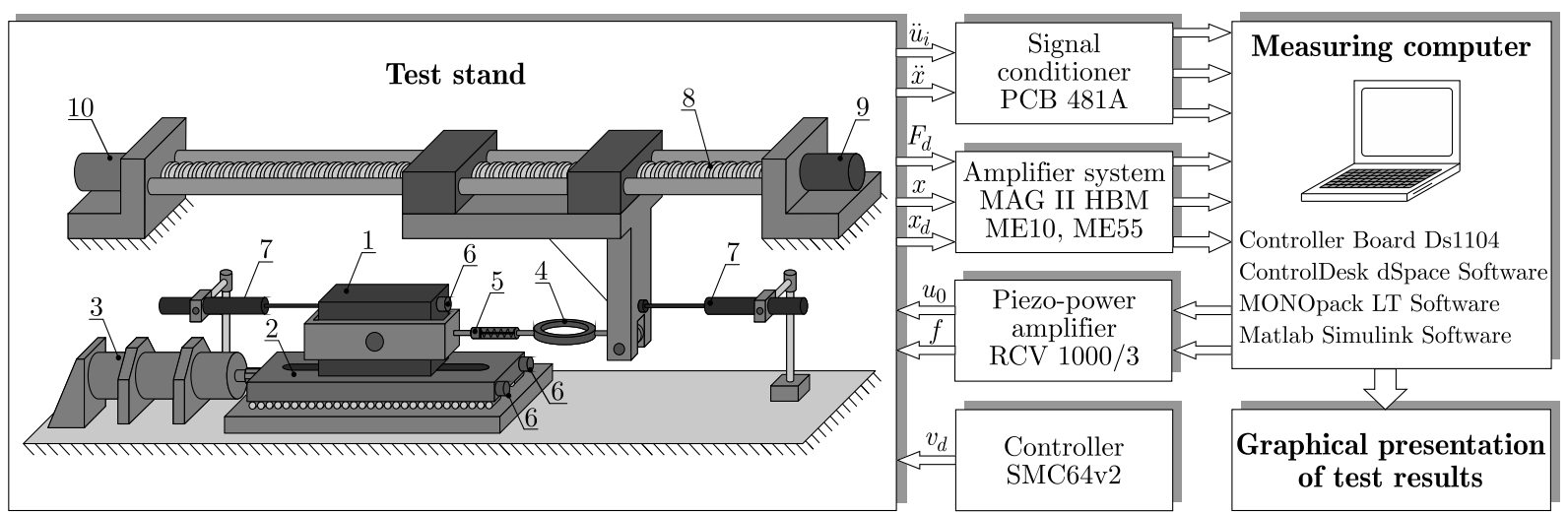

Fig. 1. The scheme of the measuring and data handling system

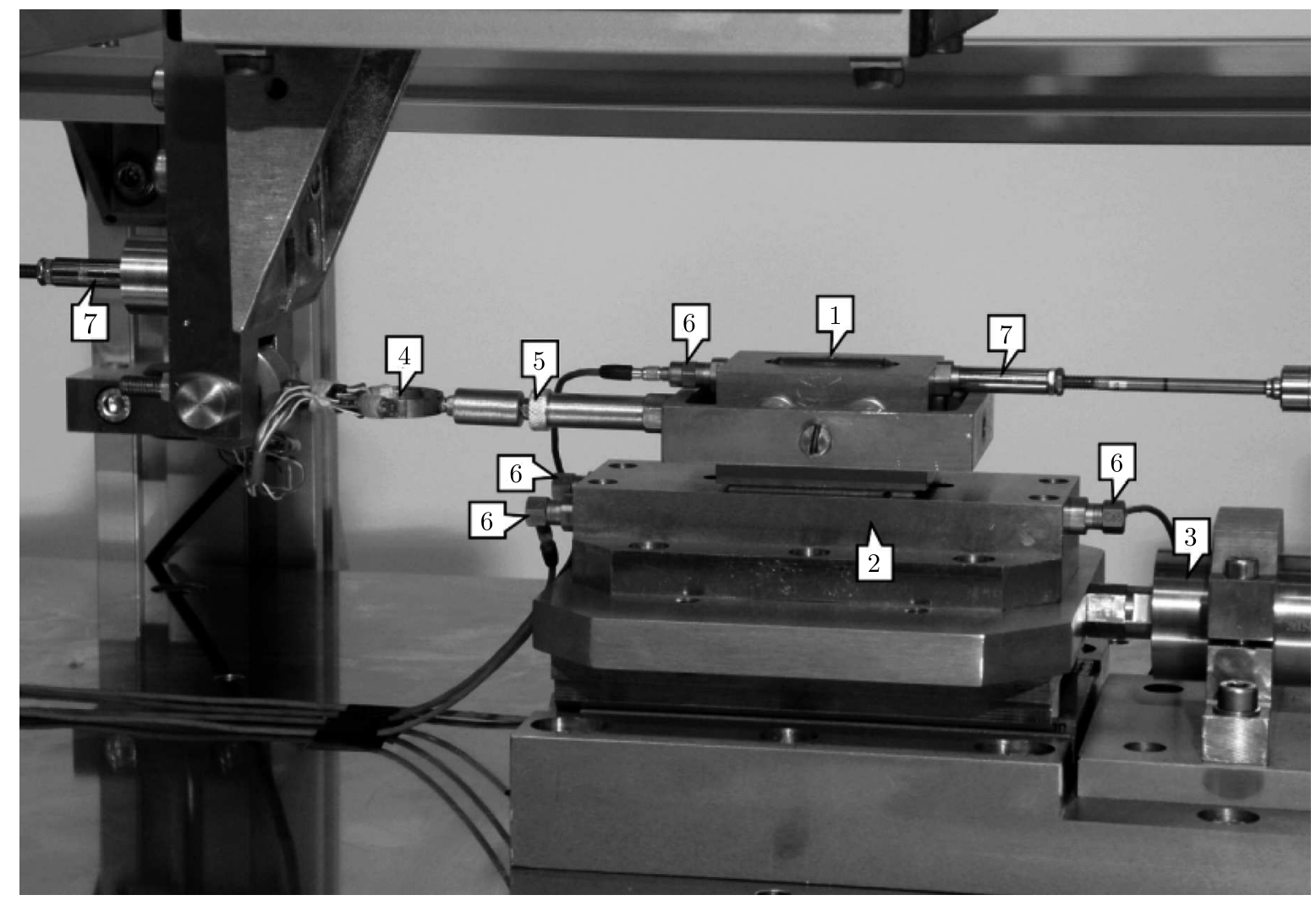

Fig. 2. A view of the mechanical part of the test stand: 1 - upper specimen, 2 - lower specimen, 3 - piezoelectric actuator, 4 - ring dynamometer, 5 - spring of specified stiffness, 6 - accelerometer, 7 - displacement sensor 


\section{Experimental investigations}

On the described stand, experimental tests of the possibility of stick-slip phenomenon reduction by forced longitudinal vibrations introduced into the substrate during upper specimen sliding at a given speed were carried out. In order to obtain stick-slip motion, the upper specimen was shifted with a suitably selected low drive velocity $v_{d}$ and at also suitably selected low stiffness of the drive $k_{d}$. The stiffness of the drive was controlled in steps by replacing the cylindrical spring (No. 5 in Fig. 1) in the drive system. Its velocity was controlled using the controller SMC64V2. The tests were carried out in the frequency range from $500 \mathrm{~Hz}$ to $4000 \mathrm{~Hz}$, with the drive velocity from $0.1 \mathrm{~mm} / \mathrm{s}$ to $2 \mathrm{~mm} / \mathrm{s}$ and with the drive stiffness ranging from $1000 \mathrm{~N} / \mathrm{m}$ to $20000 \mathrm{~N} / \mathrm{m}$. The normal pressure at contact was $p_{n}=0.03 \mathrm{~N} / \mathrm{mm}^{2}$. It was caused by the dead weight of the shifted upper specimen together with the frame $\left(F_{g}=6.52 \mathrm{~N}\right)$ and by an additional external $\operatorname{load}\left(F_{z}=30 \mathrm{~N}\right)$.

In performed tests, each series of measurements was carried out in two stages. In the first stage the substrate, the lower specimen, was at rest while the upper specimen was set into sliding motion, during which the stick-slip phenomenon occurred. In the second stage, the still motionless substrate was introduced into vibration which caused reduction of this phenomenon.

Depending on the magnitude of the amplitude of vibration velocity $v_{a}$ in relation to the drive velocity $v_{d}$, the stick-slip phenomenon was either partially reduced, which was manifested by reduction of the amplitude of changes in the driving force without changing the average value of this force, when $v_{a}<v_{d}$, or entirely eliminated, when $v_{a} \geqslant v_{d}$. In the case when $v_{a}=v_{d}$, the average value of the drive force does not change, whereas when $v_{a}>v_{d}$ it decreases.

Figures 3-5 present exemplary, experimentally determined, time characteristics of the driving force $F_{d}$ and time characteristics of displacements of the sliding specimen $x_{s}$ and the drive system $x_{d}$. In the presented experiment the following were assumed: drive velocity $v_{d}=1.0 \mathrm{~mm} / \mathrm{s}$, normal pressure at the contact $p_{n}=0.03 \mathrm{~N} / \mathrm{mm}^{2}$, stiffness of the replaceable spring in the drive system $k_{d}=10000 \mathrm{~N} / \mathrm{m}$, frequency of the forced substrate vibrations $f=1500 \mathrm{~Hz}$. The tests were carried out at four amplitudes of vibration velocity $v_{a}$ equal to: $0.2,1.1,1.5$ and $4.0 \mathrm{~mm} / \mathrm{s}$.
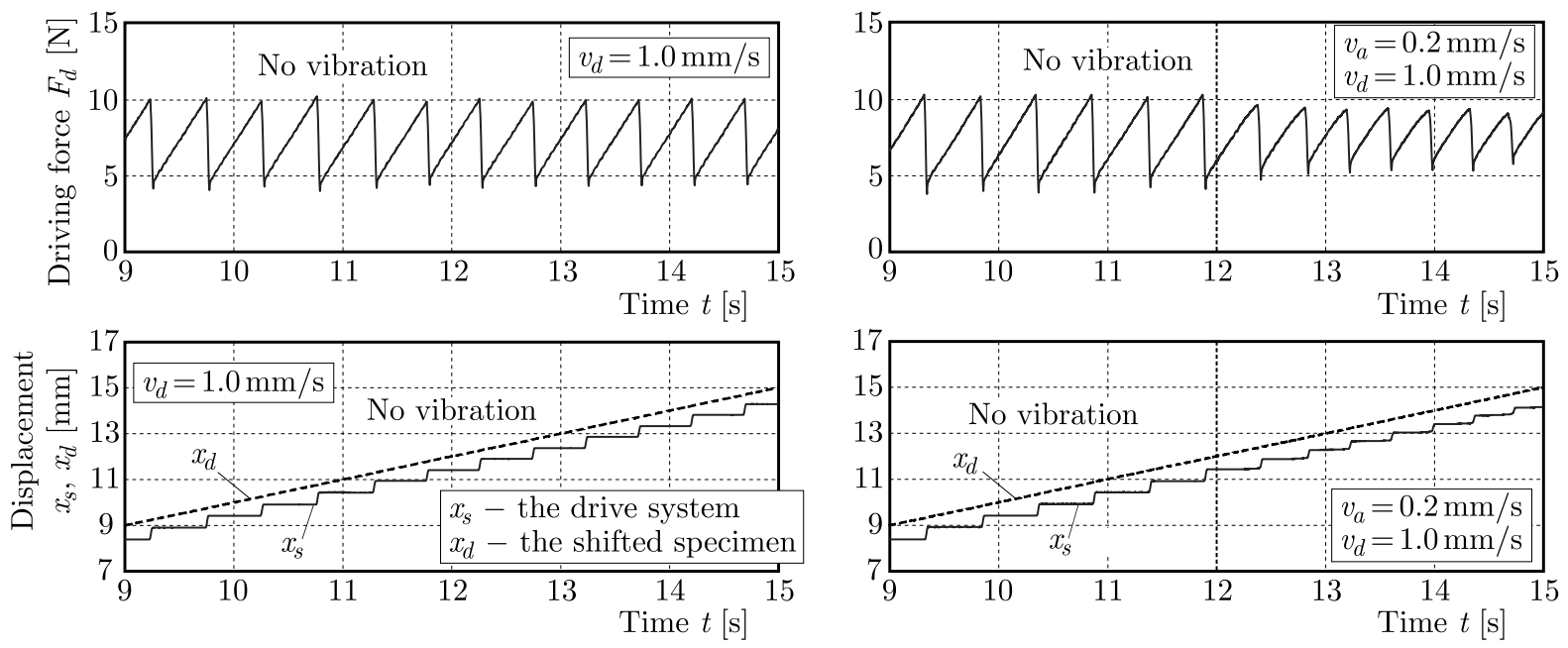

Fig. 3. Time characteristics of the driving force $F_{d}$ and displacements $x_{s}$ and $x_{d}$ determined at the drive velocity $v_{d}=1.0 \mathrm{~mm} / \mathrm{s}$ and at the condition $v_{a}<v_{d}$ 

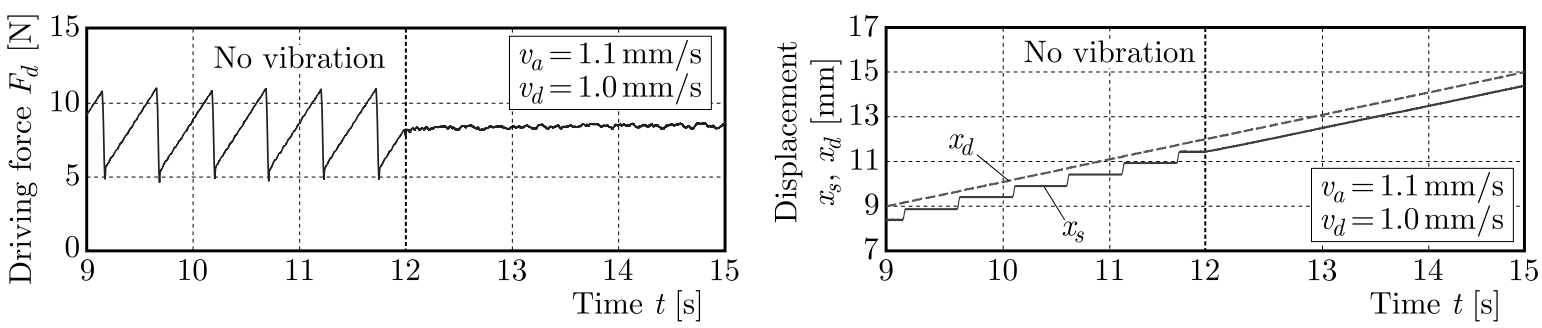

Fig. 4. Time characteristics of the driving force $F_{d}$ and displacements $x_{s}$ and $x_{d}$ determined at the drive velocity $v_{d}=1.0 \mathrm{~mm} / \mathrm{s}$ and at the condition $v_{a}=v_{d}$
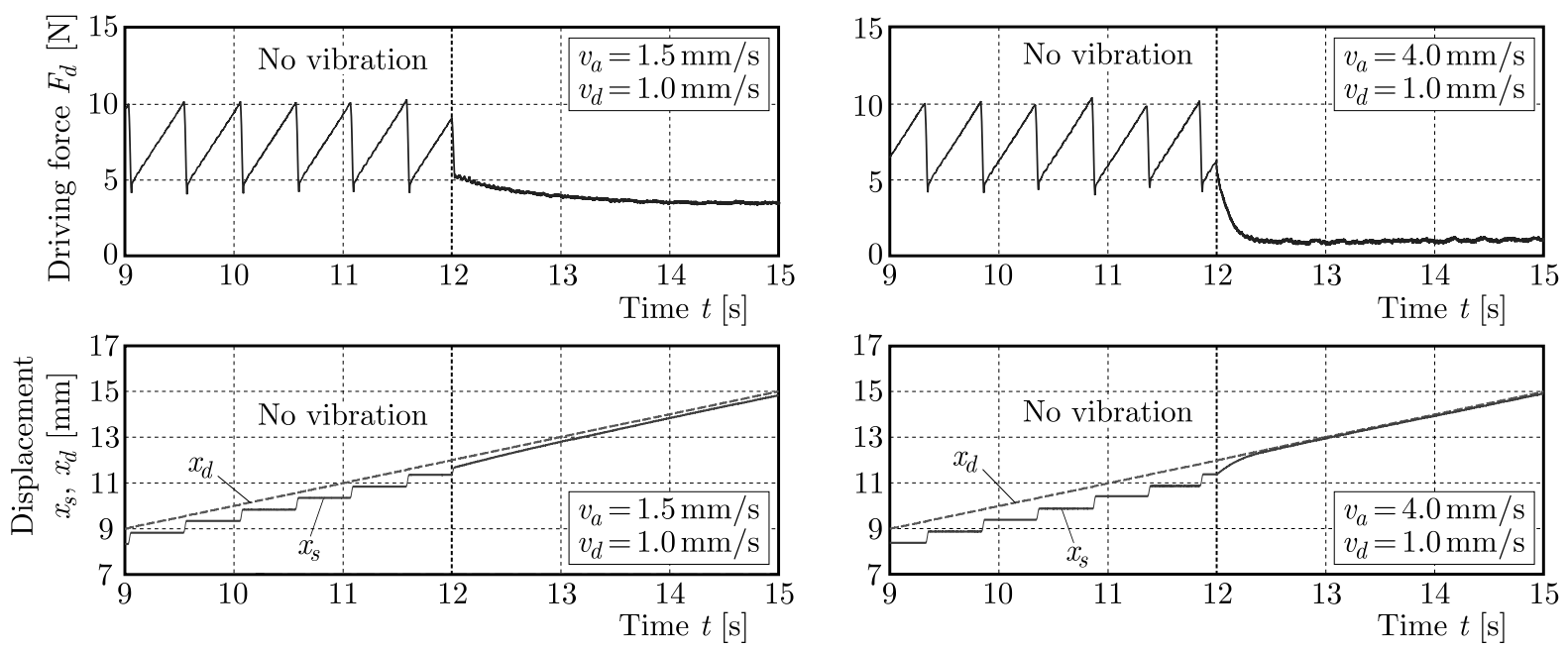

Fig. 5. Time characteristics of the driving force $F_{d}$ and displacements $x_{s}$ and $x_{d}$ determined at the drive velocity $v_{d}=1.0 \mathrm{~mm} / \mathrm{s}$ and at the condition $v_{a}>v_{d}$

\section{Computational model and its experimental verification}

In the developed model, it has been assumed that the body $A$ of mass $m$ (Fig. 6 ) is shifted over an elastic substrate $B$, which can be at any moment excited into vibration in the direction tangential to the plane of sliding and according to the shifted body motion. This body is put into sliding motion with the use of a mechanical drive. It has been assumed that the driving system can be modelled with a single, substitute, linear elastic-damping element of known stiffness $k_{d}$ and damping $h_{d}$. It has been also assumed that the driving velocity, measured at the point of the driving force $F_{d}$ application, is equal to $v_{d}$. The contact zone of the shifted body and substrate is assumed to be deformable, and both its stiffness and damping are known. The compliance characteristic of contact in the tangential direction assumed in the developed model is shown in Fig. 6b. The contact zone is modelled by a single elastic-damping substitute bar element $M N$ (Fig. 6c), in which one end, the point $N$ is rigidly connected with the shifted body, and the second one, the point $M$, is free and can freely moved over the motionless or vibrating substrate. The stiffness coefficient of contact in the tangential direction is $k_{t}$ and the damping coefficient is $h_{t}$.

The excited vibrations of the substrate are

$$
u(t)=u_{0} \sin (\omega t)
$$

where: $u_{0}$ - vibrations amplitude, and $\omega=\pi f, f$ - vibrations frequency.

The equation of motion of the shifted body has the form

$$
m \ddot{x}=F_{d}-F_{F}
$$

where: $m$-mass, $F_{d}$-driving force, $F_{F}$ - friction force. 
(a)

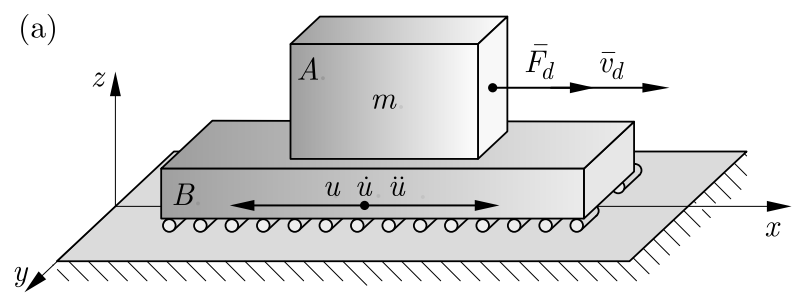

(b)

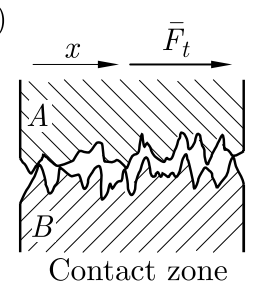

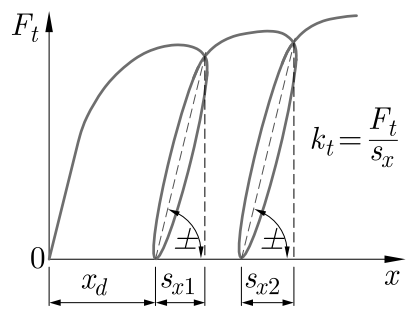

(c)

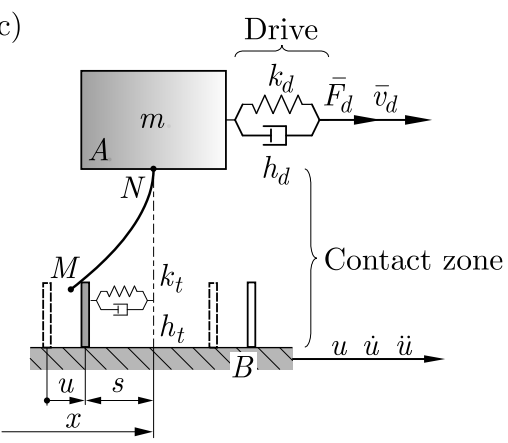

Fig. 6. Modelling of the contact zone: (a) friction pair, (b) real contact and its compliance characteristic in the tangential direction, (c) model of contact

The driving force $F_{d}$ is a sum of the two components - elastic $F_{d k}$ and dissipative $F_{d h}$

$$
F_{d}=F_{d k}+F_{d h}
$$

Their values can be determined with the use of simple dependences

$$
F_{d k}=k_{d}\left(v_{d} t-x\right) \quad F_{d h}=h_{d}\left(v_{d}-\dot{x}\right)
$$

For description of the friction force in the plane of contact of the shifted body and the vibrating substrate, the LuGre (Canudas de Wit et al., 1995) model was used. In this model, the friction force is determined according to the following dependence

$$
F_{F}=k_{t} s+h_{t} \dot{s}+h_{v} v_{r}
$$

where: $s$ - elastic deformation of contact in the direction of sliding, $h_{t}$ - damping coefficient of contact in the tangential direction, $h_{v}$ - viscous damping coefficient of contact, $v_{r}$ - relative velocity of the shifted body and substrate.

In this model, the rate $\dot{s}$ of elastic deformation of contact is described by the following relationship

$$
\dot{s}=\frac{d s}{d t}=v_{r}-\frac{\left|v_{r}\right|}{g\left(v_{r}\right)} s
$$

where

$$
v_{r}=\dot{x}-\dot{u} \quad g\left(v_{r}\right)=\frac{1}{k_{t}}\left\{F_{c}+\left(F_{s}-F_{c}\right) \exp \left[-\left(\frac{v_{r}}{v_{s}}\right)^{2}\right]\right\}
$$

where: $F_{C}$ - Coulomb friction force, $F_{s}$ - static friction force, $v_{s}$ - Stribeck velocity.

In the LuGre model, it is assumed that between the stiffness of contact $k_{t}$ and damping $h_{t}$ in the tangential direction, the following formula holds (Olsson, 1996)

$$
h_{t}=2 \zeta \sqrt{m k_{t}}
$$

where $\zeta$ - critical damping. 
In the case of sliding motion, the damping coefficient of contact depends on relative velocity $v_{r}$ and it can be described by the following equation

$$
h_{t}\left(v_{r}\right)=h_{t} \exp \left[-\left(\frac{v_{r}}{v_{d}}\right)^{2}\right]
$$

On the base of given above dependences (4.1)-(4.9), the computational model in Matlab/Simulink environment was developed for performing numerical simulation analyses of the influence of forced, tangential longitudinal vibrations of the substrate on the friction force in sliding motion and, particularly, for examination of the influence of these vibrations on the stick-slip phenomenon.

Before being used in the simulation analyzes, this model was experimentally verified by comparing the computational time characteristics of the drive force with the corresponding characteristics determined experimentally. The values of LuGre model parameters adopted in the numerical calculation are given in Table 1.

Table 1. Adopted values of the LuGre model parameters

\begin{tabular}{|c|c|}
\hline$k_{t}$ & $71.318 \cdot 10^{6} \mathrm{~N} / \mathrm{m}$ \\
\hline$h_{t}$ & $1.377 \cdot 10^{4} \mathrm{Ns} / \mathrm{m}$ \\
\hline$h_{v}$ & $0 \mathrm{Ns} / \mathrm{m}$ \\
\hline$F_{c}$ & $8.3 \mathrm{~N}$ \\
\hline$F_{s}$ & $10.8-12.2 \mathrm{~N}$ \\
\hline$v_{s}$ & $0.00035 \mathrm{~m} / \mathrm{s}$ \\
\hline
\end{tabular}

The contact stiffness $k_{t}$ value was determined experimentally (Leus and Gutowski, 2009). The damping coefficient $h_{t}$ was determined from formula (4.8) assuming $\zeta=1$ according to Olsson (1996). For dry contact, viscous damping $h_{v}=0 \mathrm{Ns} / \mathrm{m}$ was assumed. The value of the force $F_{c}$ is proportional to the normal pressure of contact and the coefficient of static friction, whose value was determined experimentally. The values of force $F_{s}$ and velocity $v_{s}$ were determined during model verification. This verification was carried out at chosen stiffnesses and velocities of the drive, at chosen normal pressure and selected parameters of forced vibrations of the base. Exemplary, comparative drafts of the driving force at driving velocity $v_{d}$ equal to $0.5 \mathrm{~mm} / \mathrm{s}$, for three values of drive stiffness: $k_{d}=5950 \mathrm{~N} / \mathrm{m}, 11700 \mathrm{~N} / \mathrm{m}, 17900 \mathrm{~N} / \mathrm{m}$, at normal pressure $p_{n}=0.03 \mathrm{~N} / \mathrm{mm}^{2}$, and various amplitudes of vibration velocities: $v_{a}=0.2,0.3,0.5,0.6,1.0,1.1$, 2.8 and $3.5 \mathrm{~mm} / \mathrm{s}$ are given in Figs. 7-9.

It is seen that at any analyzed case, an excellent consistency of experimentally determined results with those calculated with the use of the developed model is obtained. It is seen that the developed model reflects very well both the stick-slip motion and its changes caused by forced vibrations introduced into the contact area, and further, after elimination of the stick-slip motion, the changes of the friction force value. This testifies about correctness of the elaborated model and about possibility of its use in numerical analyses of the influence of longitudinal tangential vibrations on the friction force in sliding motion and on the stick-slip phenomenon.

\section{Simulating analyses}

The main purpose of the conducted simulation analyzes was to examine the conditions of complete elimination or partial reduction of the stick-slip phenomenon in sliding motion at the given drive velocity and drive stiffness by introducing longitudinal tangential vibration of high frequency into the contact zone between the shifted body and substrate. The second purpose was determination of the drive force changes with a further increase in the amplitude of forced 
(a)

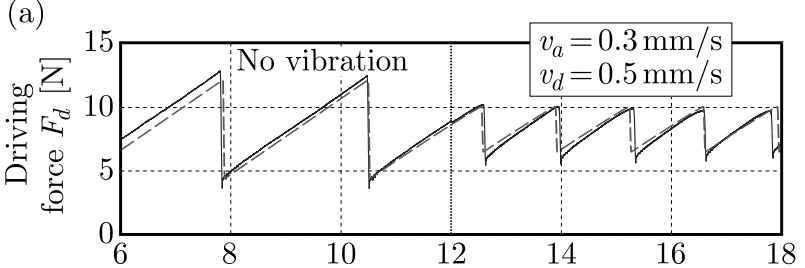

(c)

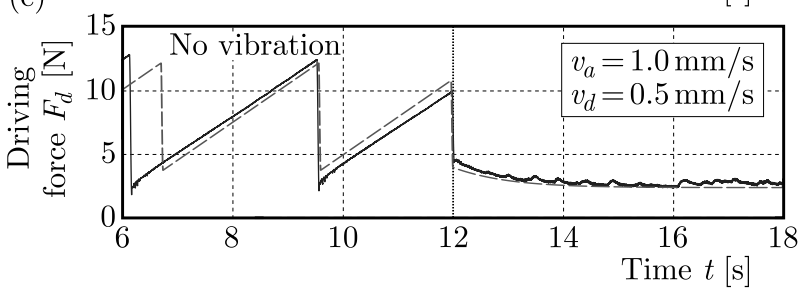

(b)

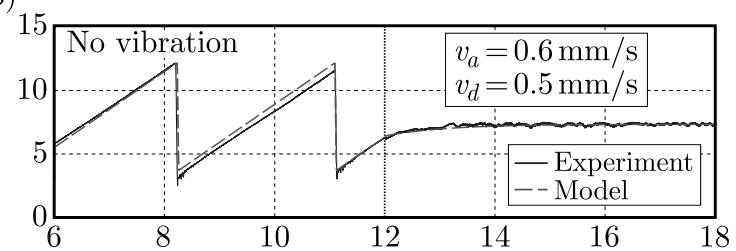

(d)

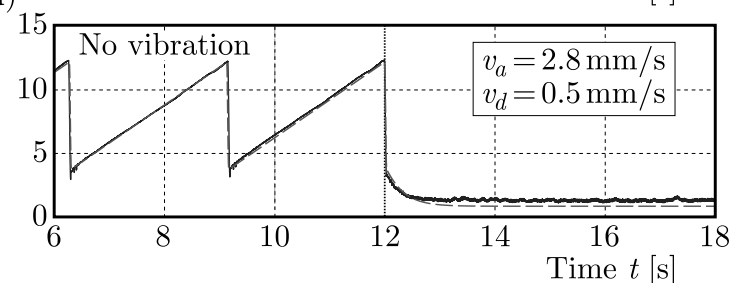

Fig. 7. Comparison of model and experimentally determined time characteristics of the driving force in sliding motion at drive velocity $v_{d}=0.5 \mathrm{~mm} / \mathrm{s}$ and stiffness $k_{d}=5950 \mathrm{~N} / \mathrm{m}$ for various parameters of

forced vibrations: (a) $v_{a}=0.3 \mathrm{~mm} / \mathrm{s}$, (b) $v_{a}=0.6 \mathrm{~mm} / \mathrm{s}$, (c) $v_{a}=1.0 \mathrm{~mm} / \mathrm{s}$, (d) $v_{a}=2.8 \mathrm{~mm} / \mathrm{s}$

(a)

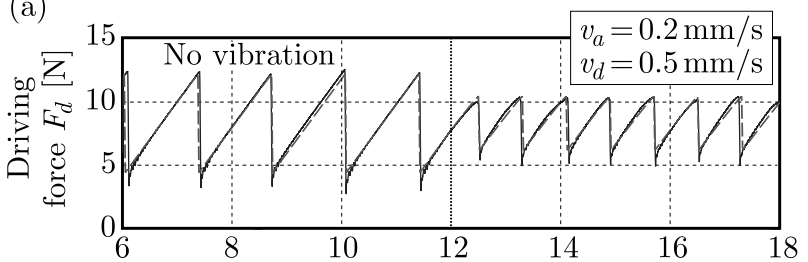

(c)

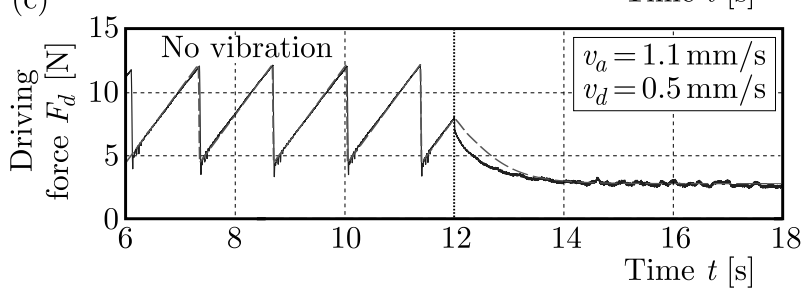

(b)

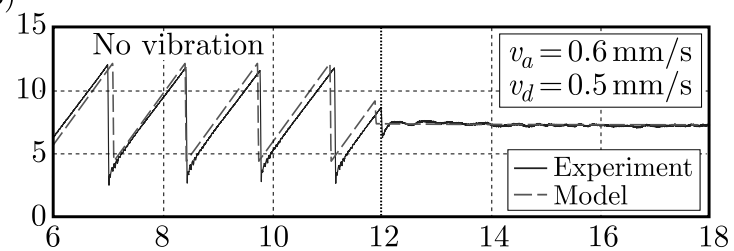

(d)

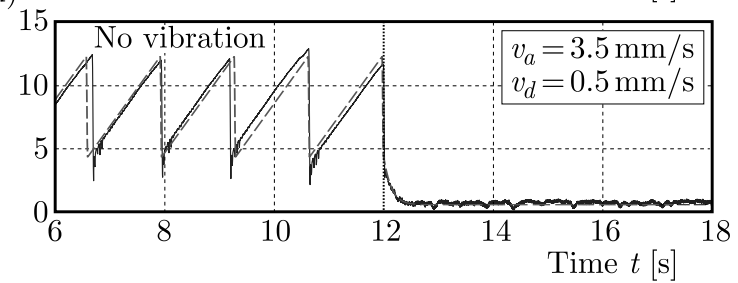

Fig. 8. Comparison of model and experimentally determined time characteristics of the driving force in sliding motion at drive velocity $v_{d}=0.5 \mathrm{~mm} / \mathrm{s}$ and stiffness $k_{d}=11700 \mathrm{~N} / \mathrm{m}$ for various parameters of

forced vibrations: (a) $v_{a}=0.2 \mathrm{~mm} / \mathrm{s}$, (b) $v_{a}=0.6 \mathrm{~mm} / \mathrm{s}$, (c) $v_{a}=1.1 \mathrm{~mm} / \mathrm{s}$, (d) $v_{a}=3.5 \mathrm{~mm} / \mathrm{s}$

(a)

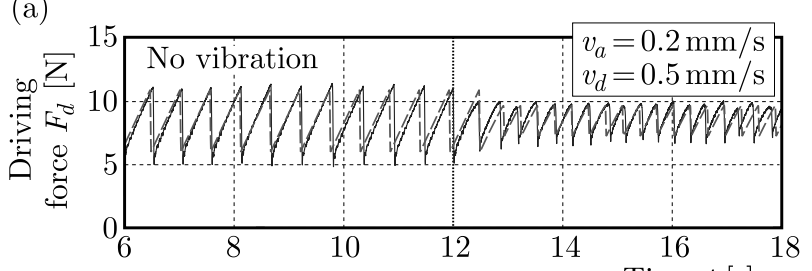

(c)

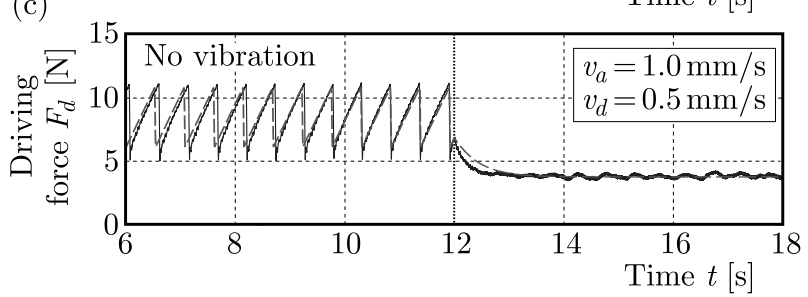

(b)

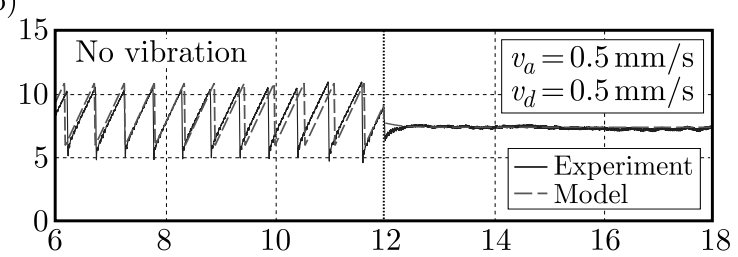

(d)

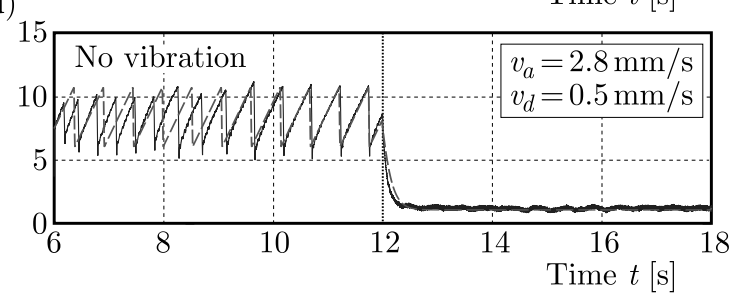

Fig. 9. Comparison of model and experimentally determined time characteristics of the driving force in sliding motion at drive velocity $v_{d}=0.5 \mathrm{~mm} / \mathrm{s}$ and stiffness $k_{d}=17900 \mathrm{~N} / \mathrm{m}$ for various parameters of forced vibrations: (a) $v_{a}=0.2 \mathrm{~mm} / \mathrm{s}$, (b) $v_{a}=0.5 \mathrm{~mm} / \mathrm{s}$, (c) $v_{a}=1.0 \mathrm{~mm} / \mathrm{s}$, (d) $v_{a}=2.8 \mathrm{~mm} / \mathrm{s}$ 
vibration velocity after elimination of the stick-slip motion. The analyzes were carried out in the drive velocity range $0.5 \leqslant v_{d} \leqslant 1.5 \mathrm{~mm} / \mathrm{s}$ and for drive stiffness equal to $k_{d}=11700 \mathrm{~N} / \mathrm{m}$.

Figure 10 illustrates the changes of the stick-slip reduction level, determined in numerical analyzes carried out using the developed model, as a function of the amplitude of forced vibration velocity related to the drive velocity $v_{a} / v_{d}$. As a measure of the stick-slip reduction level, in preformed analyses, the value of coefficient $r$ defined as

$$
r=\frac{\Delta F_{s d}}{\Delta F_{s s}}
$$

is taken. In this dependence, $\Delta F_{s d}$ is the current amplitude of the drive force during sliding on the vibration base, and $\Delta F_{s s}$ is the amplitude of the drive force during sliding on the motionless substrate.

To compare, the results obtained in the experimental tests are also marked on the graphs. It is seen that their compatibility with the results of simulation analysis is very good.

(a)

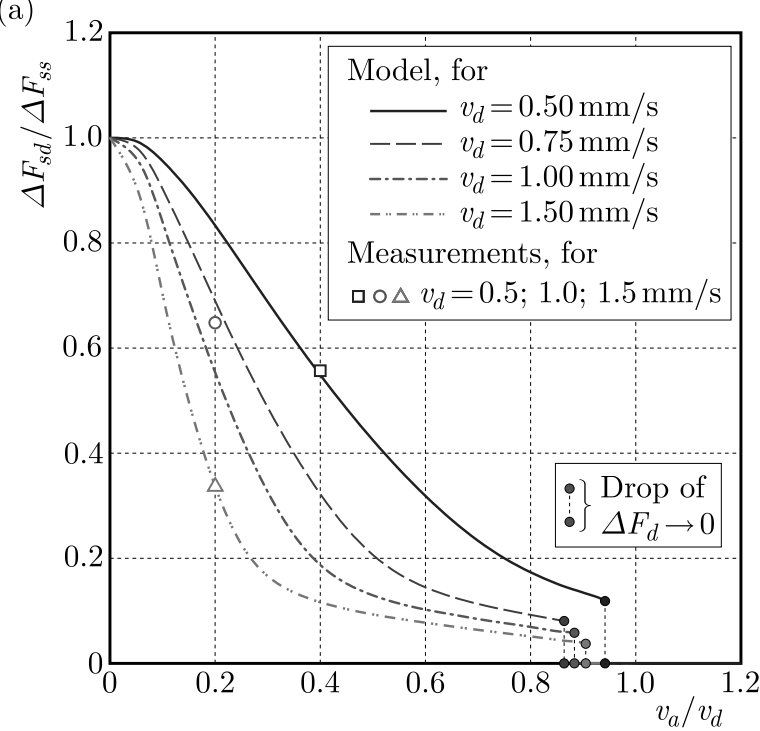

(b)

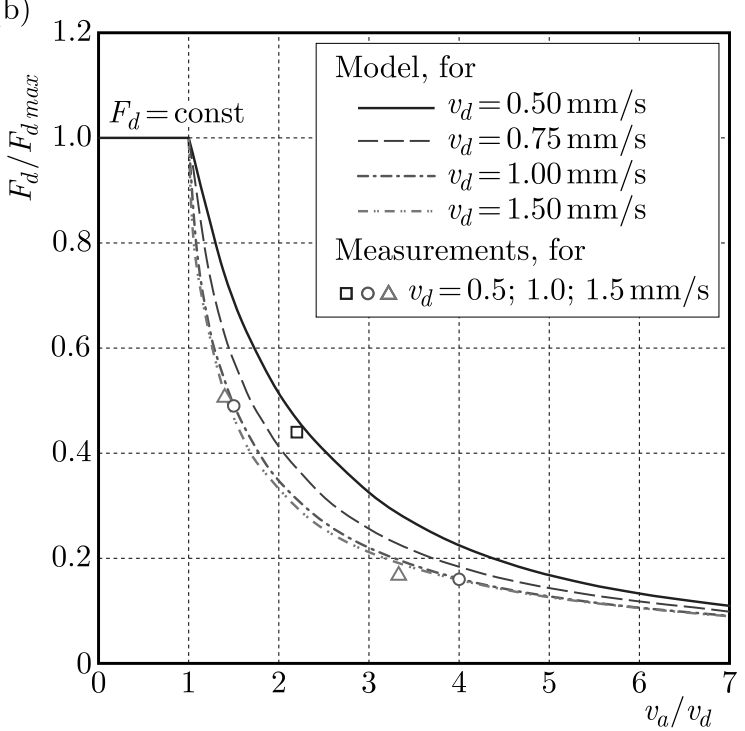

Fig. 10. Impact of forced vibrations on the stick-slip motion: (a) reduction of stick-slip vibrations under the effect of forced longitudinal vibration at $v_{a}<v_{d}$ and their elimination at $v_{a} \approx v_{d}$, (b) reduction of the drive force under the effect of forced vibration of the substrate at $v_{a}>v_{d}, f=1500 \mathrm{~Hz}$

The graphs illustrate that the influence of longitudinal vibration on the friction processes in the contact zone between the shifted body and base should be considered in two neighboring intervals of vibration parameters, closely related to the current slip velocity. In the first interval, when the vibration parameters are such that the amplitude of vibration velocity $v_{a}$ is smaller than the drive velocity $\left(v_{a}<v_{d}\right)$, the stick-slip vibrations are reduced, their amplitude and sticking period decrease but the average value of the friction force remains unchanged. When the amplitude of vibration velocity is grater than the speed of drive $\left(v_{a}>v_{d}\right)$, the stick-slip motion is entirely eliminated. With a further increase in the amplitude of vibration velocity, the average value of the friction force decreases.

The amplitude of vibration velocity $v_{a}$ in tangential oscillations, described by the dependence $u=u_{0} \sin (\omega t)$, is a function of both the vibration amplitude $u_{0}$ and their angular velocity $\omega$, and it can be described by the following relationship

$$
v_{a}=u_{0} \omega=2 \pi f u_{0}
$$

The modification of this amplitude can be achieved either by changing the values of both $f$ and $u_{0}$ parameters simultaneously, or by changing the value of one parameter at the fixed value 
of the second one. However, the particular impact of these two parameters on the effectiveness of stick-slip reduction is different. It is clearly seen from the graphs presented in Figs. 11a and 11b. The first graph presents the course of the level of stick-slip vibration reduction as a function of the amplitude of forced vibrations $u_{0}$ of the substrate at a given frequency $f$, while the second one presents the course of the level of stick-slip vibration reduction as a function of frequency $f$ of forced vibrations at a fixed amplitude $u_{0}$ of these vibrations.

(a)

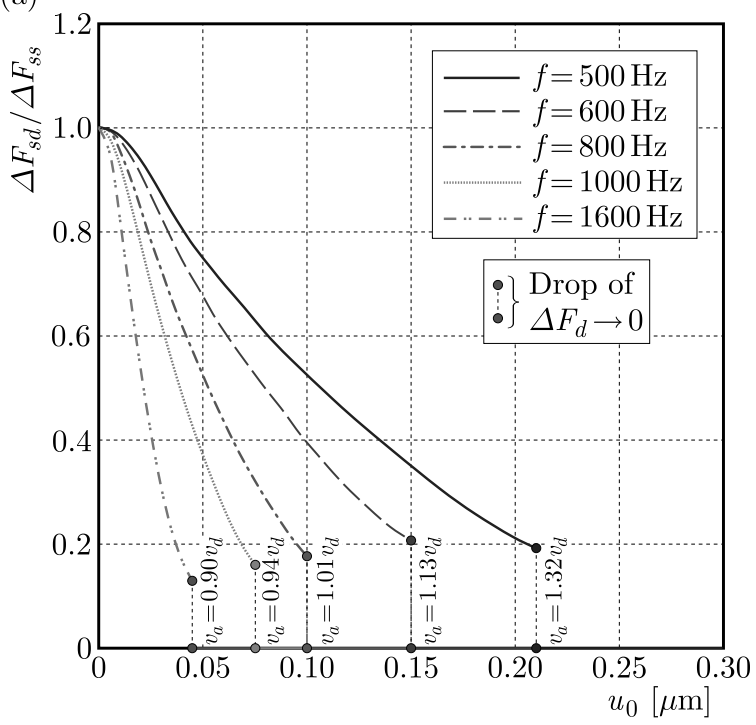

(b)

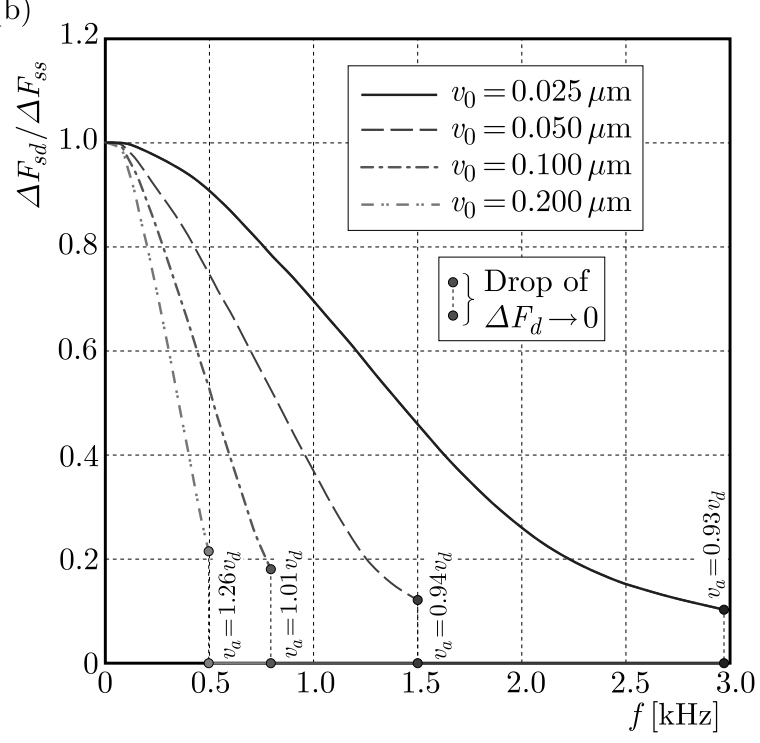

Fig. 11. The dependence of level of stick-slip vibration reduction on: (a) the amplitude $u_{0}$ of forced vibrations of the substrate at a fixed frequency $f$ of forced vibrations and (b) the frequency $f$ of forced vibrations of the substrate at a fixed amplitude $u_{0}$ of forced vibrations; drive velocity $v_{d}=0.5 \mathrm{~mm} / \mathrm{s}$

From the graphs presented in Fig. 11, it can be seen that at a fixed value of one of the forced vibration parameters, i.e. the amplitude $u_{0}$ or frequency $f$, there is a limit value of the second parameter after exceeding whose stick-slip motion, at the given drive velocity, is entirely eliminated. This limit value is related to the drive velocity $v_{d}$ and the amplitude of vibration velocity $v_{a}$. However, it can be seen that at low frequencies and large amplitudes of forced vibrations, the fulfillment of the condition $v_{a} / v_{d}>1$ does not guarantee complete elimination of the stick-slip motion.

\section{Conclusion}

Experimental tests and simulation analyzes indicate that the introduction into the contact zone between the sliding body and the substrate longitudinal tangential vibration can not only reduce, but also completely eliminate the sick-slip phenomenon which appears in sliding motion at low velocity and low drive stiffness. The stick-slip reduction is manifested by reduction in the vibration amplitude. The degree of this reduction depends on the amplitude of velocity of forced vibration in relation to the drive velocity. When this amplitude exceeds the drive velocity, the stick-slip phenomenon can be entirely eliminated, whereas when the amplitude increases further, the average friction force is reduced.

An excellent consistency of simulation and experimental tests results proved the correctness of the elaborated model, thus it can be used to analyze the impact of forced vibration of the substrate on stick-slip motion. 


\section{References}

1. Aarsnes U.J.F., Di Meglio F., Shor R.J., 2018, Avoiding stick slip vibrations in drilling through startup trajectory design, Journal of Process Control, 70, 24-35

2. Abdo J., Tahat M., Abouelsoud A., 2009, The effect of excitation frequencies on stick-slip amplitude, 3rd International Conference on Integrity, Reliability and Failure, Porto, Portugal, 2024 July

3. Abdo J., Mahmoud T., Abouelsoud A., Danish M., 2010, The effect of frequency of vibration and humidity on stick-slip amplitude, International Journal of Mechanics and Materials in Design, 6, 45-51

4. Abdo J., ZAIER R., 2012, A novel pin-on-disk machine for stick-slip measurements, Materials and Manufacturing Processes, 27, 751-755

5. Åstrom K.J., Canudas de Wit C., 2008, Revisiting the LuGre friction model, IEEE Control Systems, 28, 6, 101-114

6. Broniec Z., Lenkiewicz W., 1982, Static friction process under dynamic loads and vibration, Wear, 80, 261-271

7. Canudas de Wit C., Olsson H., Astrom K.J., Lischinsky P., 1995, A new model for control of systems with friction, IEEE Transactions on Automatic Control, 40, 3, 419-425

8. Haessig D.A., Friedland B., 1991, On the modeling and simulation of friction, ASME Journal of Dynamic Systems, Measuremant and Control, 113, 3, 354-362

9. JuRCZYŃski Ł., 2013, The use of high frequency forced vibrations for elimination of stick-slip phenomenon (in Polish), Master thesis, West Pomeranian Uniwersity of Technology, Szczecin

10. Kligerman Y., Varenberg M., 2014, Elimination of stick-slip motion in sliding of split or rough surface, Tribology Letters, 53, 2, 395-399

11. Kröger M., Neubauer M., Popp K., 2008, Experimental investigation on the avoidance of self-excited vibrations, Philosophical Transactions of the Royal Society A, 366, 1866, 785-810

12. LENKIEWICZ W., 1969, The sliding friction process effect of external vibration, Wear, 13, 2, 99-108

13. Leus M., Abrahamowicz M., 2019, Experimental investigations of elimination the stick-slip phenomenon in the presence of longitudinal tangential vibration, ACTA Mechanica et Automatica, 13, $1,45-50$

14. Leus M., Gutowski P., 2009, The experimental analysis of the tangential stiffness of the flat contact joints (in Polish), Modelowanie Inżynierskie, 37, 185-192

15. Mfoumou G.S., Kenmoé G.D., Kofané T.C., 2019, Computational algorithms of time series for stick-slip dynamics and time-delayed feedback control of chaos for a class of discontinuous friction systems, Mechanical Systems and Signal Processing, 119, 399-419

16. Neubauer M., Neuber C.-C., Popp K., 2005, Control of stick-slip vibrations, Solid Mechanics and its Applications, 130, 223-232

17. Olsson H., 1996, Control Systems with Friction, Lund

18. Popov V.L., Starcevic J., Filippov A.E., 2010, Influence of ultrasonic in-plane oscillations on static and sliding friction and intrinsic length scale of dry friction processes, Tribology Letters, 30, $1,25-30$

19. Popp K., Rudolph M., 2003, Avoidance of stick-slip motion by vibration control, Proceedings in Applied Mathematics and Mechanics, 3, 120-121

20. Popp K., Rudolph M., 2004, Vibration control to avoid stick-slip motion, Journal of Vibration and Control, 10, 1585-1600

21. QIU H., YANG J., ButT S., 2018, Investigation on bit stick-slip vibration with random friction coefficients, Journal of Petroleum Science and Engineering, 164, 127-139 
22. Rymuza Z., 1989, Sliding guides in small mechanisms and precision instruments - tribological issues (in Polish), Pomiary, Automatyka, Kontrola, 5

23. Rymuza Z., 1992, The stick-slip phenomenon (in Polish), Pomiary, Automatyka, Kontrola, 12, 290-295

24. Teidelt E., Starcevic J., Popov V.L., 2012, Influence of ultrasonic oscillation on static and sliding friction, Tribology Letters, 48, 1, 51-62

25. Tolstoi D.M., 1967, Significance of the normal degree of freedom and natural normal vibrations in contact friction, Wear, 10, 199-213

26. Tolstoi D.M., Borisova G.A., Griegorova S.R., 1973, Friction reduction by perpendicular oscillation, Doklady Technical Physis, 17, 9, 907-909

27. ZHU X., TANG L., YANG Q., 2014, A literature review of approaches for stick-slip vibration suppression in oilwell drillstring, Advances in Mechanical Engineering, 2014, 1-17

28. Zuleeg J., 2015, How to measure, prevent, and eliminate stick-slip and noise generation with lubricants, SAE Technical Paper, 2015-01-2259, 1-7

Manuscript received August 13, 2019; accepted for print November 5, 2019 